\title{
Special issue on PODC 2015 and PODC 2016
}

Hagit Attiya ${ }^{1}$

Published online: 29 October 2019

๑) Springer-Verlag GmbH Germany, part of Springer Nature 2019

This special issue of Distributed Computing is based on papers that originally appeared as extended abstracts in the Proceedings of the 34rd Symposium on Principles of distributed computing (PODC 2015), held in Donostia-San Sebastián, Spain, and in the Proceedings of the 35rd Symposium on Principles of distributed computing (PODC 2016), held in Chicago, Illinois, USA. It also include a paper invited to the special issue of PODC 2012.

The papers for the Special Issue were chosen by the respective Program Committees from the regular papers presented at the symposium, based on their quality and representation of the spectrum of topics encompassed by the
Symposium. In addition to being reviewed, in preliminary form, by the Program Committee, the full papers submitted for the Special Issue were refereed according to the standard practices of Distributed Computing. We thank the handling editors, the referees and the authors of these papers.

Publisher's Note Springer Nature remains neutral with regard to jurisdictional claims in published maps and institutional affiliations.
Hagit Attiya

hagit@cs.technion.ac.il

1 Department of Computer Science, Technion, Haifa, Israel 